\title{
Coupled Multibody Model Of Industrial Robot With Milling Simulator For Trajectory Compensation
}

\author{
V. Dambly ${ }^{1}$, H.N. Huynh ${ }^{2}$, O. Verlinden ${ }^{1}$, É. Rivière-Lorphèvre ${ }^{1}$ \\ ${ }^{1}$ Faculty of Engineering \\ University of Mons \\ 20 place du Parc, 7000 Mons, Belgium \\ [valentin.dambly,olivier.verlinden, \\ edouard.rivierelorphevre]@umons.ac.be \\ ${ }^{2}$ Department of Mechanical Engineering \\ University of British Columbia \\ Vancouver BC V6T 1Z4, Canada \\ hoainam.huynh@ubc.ca
}

\begin{abstract}
Robotic machining is a fast-growing technology in the field of mechanical manufacturing. Indeed, it is generally accepted that for the same working space, a fully equipped robotic machining cell can cost 30 to $50 \%$ less than a conventional machine tool. However, inaccuracies resulting either from vibrations or deflections occur while the robot is subjected to cutting forces, inherent to its flexible structure. As an order of magnitude, the stiffness at the tool-tip is about $1 \mathrm{~N} / \mu \mathrm{m}$ for industrial robots against more than $50 \mathrm{~N} / \mu \mathrm{m}$ for $\mathrm{CNC}$ machine tools. The flexibility source has been investigated and appears to be caused by the robot articulations in a proportion of $80 \%$ while the remaining flexibility issues from the structural elasticity. In order to improve the accuracy of robotic machining operations, several approaches have been carried out such as the study of stable cutting conditions and the online/offline compensation of the tool trajectory.

Two aspects of the operation must be modeled, on the one hand the model of the cutting machine, being an industrial robot in robotic machining, and on the other hand, the machining model including the resulting geometry of the workpiece. A coupled model is then proposed with the multi-body model of the robot subjected to machining forces. The multi-body model includes the flexibility induced by the structure and the articulations. In order to compensate the deviations, a solution is proposed where the trajectory is discretized in nodes with a compensation taking the system dynamics into account by successive simulations of the operation. The algorithm involves two steps, firstly it aims to detect critical locations of the path and add or reposition nodes to reduce the deviation and secondly an optimization layer modifies nodes positions and velocities for a finer reduction. The method is deployed for three systems of increasing complexity for a face milling operation, showing a machining error reduction.
\end{abstract}

Keywords: Robotics, dynamic simulation, trajectory optimization, coupled models.

\section{INTRODUCTION}

Robotic machining is a fast-growing technology in the field of mechanical manufacturing. Indeed, it is generally accepted that for the same working space, a fully equipped robotic machining cell can cost 30 to $50 \%$ less than a conventional machine tool. Furthermore, robotic machining enables an interesting agility in the cutter motion to deal with complex workpieces geometry. However, inaccuracies resulting either from vibrations or deflections occur while the robot is subjected to cutting forces. As an order of magnitude, the stiffness at the tool-tip is about $1 \mathrm{~N} / \mu \mathrm{m}$ for industrial robots against more than $50 \mathrm{~N} / \mu \mathrm{m}$ for CNC machine tools [1].

The causes of these issues are numerous and have been identified then classified according to their nature [2]. Among the deviations sources, a major contribution appears to be the flexibility of the 
robot, caused by its articulations in a proportion of $80 \%$ while the remaining flexibility issues from the structural elasticity of the links [3].

In order to improve the accuracy of robotic machining operations, several approaches have been carried out such as the study of stable cutting conditions and the online/offline compensation of the tool trajectory [2]. Within the frame of industry 4.0 and the concept of virtual twin, offline models can be developed in order to predict instabilities and compensate deviations. However, for the offline compensation, it is necessary to model both aspects of the operation, on the one hand the model of the cutting machine, being an industrial robot in robotic machining, and on the other hand, the machining model including the resulting geometry of the workpiece [4]. Offline compensation methods are closely related to modeling of the robot flexibility. The static deflection is directly computed from the identified stiffness matrix and the estimation of the milling forces.

The existing models mostly locate the flexibility at the articulations (precisely in the gearbox and bearings) and at links (presenting significant bending depending on their shape). Several offline compensation approaches are developed in the literature depending on the parameters chosen for the flexibility modeling [2]. These approaches propose to determine an equivalent torsional stiffness value at the articulation and to use it to calculate the corresponding deflection of the tool center point $[5,6]$.

The more sophisticated the flexibility modeling the better the static deflection evaluation, with for example a complete identification at the articulation level [5] or with simpler torsional model such as the virtual joint modeling approach [7]. In each case, an equivalent stiffness value is computed (with for example the Conservative Congruence Transformation [8]) and used to calculate the corresponding static deflection of the tool center point [1]. This deflection is used to shift the initial trajectory, also called the mirroring method [9].

Even though the stiffness-based deviation estimation presents a important error reduction, the dynamic deflection has not been addressed [4] and may cause non-negligible deviations. The dynamical behavior is significant at different moments of the operation for example when the tool enters or leaves the matter or even while performing small amplitude back and forth movements (changing the direction of motors torques) [10].

The optimal trajectory generation for underactuated flexible robots is an active research topic where optimal trajectory generation and control are central issues [11]. However, the perturbations generated by machining forces make these algorithms complex to apply in our context. An other reason requiring dynamic models is the presence of damping. It has indeed been shown that the articular flexibility modeling is improved by adding a damping contribution [3].

A robot performing a machining operation can be considered as a multibody chain with perturbation forces applied on the tool center point (TCP). In order to be able to simulate 5-axis operations, it is necessary to include a machining force module that can compute these forces from the tool motion. Concerning the modeling of machining operation, several approaches exist depending on the expected inputs of the simulation [12]. The most appropriate candidates for time-based simulation of 5-axis operations where the cutting forces and workpiece geometry are needed at each time-step are the voxel and the dexel approaches [12]. Models based on voxels have been developed for model-based compensation [6]. However, the complexity of voxel model is up to $O\left(n^{3}\right)$ against $O\left(n^{2}\right)$ for dexel. Besides, for 2.5D operations, the stack of slices approach is faster and reliable [13].

This paper first describes the flexibility model of the robot. Afterwards, the repositioning algorithm is presented and applied on several multibody systems. Three models of increasing complexity are addressed. In the first place, a minimal case, being the tool-mass body fixed with linear spring/damper to a support whose motion is prescribed, is studied. Afterwards, non-linear robots 
are a two-degree-of-freedom robot and the machining robot Stäubli TX200, with the introduction of torsional spring/damper pairs at the articulations to model the flexibility. The repositioning of the trajectory nodes is improved with an optimization layer considering nodes positions and velocities as design variables. Perspectives are discussed for the development, refinement and extension for 5-axis operations of the proposed method.

\section{MULTIBODY DYNAMIC MODEL}

The equations of motions for a multiple-degree-of-freedom mechanical system are expressed as follows

$$
\mathbf{M}_{\mathbf{q}}(\mathrm{q}) \ddot{\mathbf{q}}(\mathbf{t})+\mathbf{h}_{\mathbf{q}}(\mathrm{q}, \dot{\mathrm{q}})=\mathbf{F}(\mathbf{t})
$$

where $\mathbf{M}_{\mathbf{q}}(\mathrm{q}), \mathbf{h}_{\mathbf{q}}(\mathrm{q}, \dot{\mathrm{q}})$ and $\mathbf{F}(\mathbf{t})$ are defined in generalized coordinates, according to the virtual power theorem, with equations $(2,3,4)$, where $\mathbf{M}_{\mathbf{q}}(\mathbf{q})$ the mass matrix, $\mathbf{h}_{\mathbf{q}}(\mathrm{q}, \dot{\mathrm{q}})$ the vector gathering the Coriolis, gyroscopic and centrifugal forces and $\mathbf{F}(\mathbf{t})$ the vector of external forces applied on the system are expressed in terms of the generalized coordinates $q$ according to equations (2) to (4). The vector $\mathrm{q}$ regroups the actuated degrees-of-freedom $\mathrm{q}_{a}$ and the unactuated degrees-of-freedom $\mathrm{q}_{u}$.

$$
\begin{gathered}
\mathbf{M}_{\mathrm{q}}(\mathbf{q})=\sum_{i=1}^{n_{\mathrm{B}}}\left(m_{i}\left[\mathbf{J}_{S, i}\right]_{\text {Base }}^{T} \cdot\left[\mathbf{J}_{S, i}\right]_{\text {Base }}+\left[\mathbf{J}_{\omega, i}\right]_{\text {Base }}^{T} \cdot \mathbf{R}_{\text {Base }, i} \cdot\left[\Phi_{G, i}\right]_{i} \cdot \mathbf{R}_{\text {Base }, i}^{T} \cdot\left[\mathbf{J}_{\omega, i}\right]_{\text {Base }}\right) \\
\mathbf{h}_{\mathrm{q}}(\mathrm{q}, \dot{\mathrm{q}})=\sum_{i=1}^{n_{\mathrm{B}}}\left(m_{i}\left[\mathbf{J}_{S, i}\right]_{\text {Base }}^{T} \cdot\left[\dot{\mathbf{J}}_{S, i}\right]_{\text {Base }}+\left[\mathbf{J}_{\omega, i,}\right]_{\text {Base }}^{T} \cdot \mathbf{R}_{\text {Base }, i} \cdot\left[\Phi_{G, i}\right]_{i} \cdot \mathbf{R}_{\text {Base }, i}^{T} \cdot\left[\dot{\mathbf{J}}_{\omega, i}\right]_{\text {Base }}\right) \dot{\mathrm{q}} \\
+\left[\mathbf{J}_{\omega, i}\right]_{\text {Base }}^{T} \cdot\left(\left\{\omega_{i}\right\}_{\text {Base }} \times \mathbf{R}_{\text {Base }, i} \cdot\left[\Phi_{G, i}\right]_{i} \cdot \mathbf{R}_{\text {Base }, i}^{T} \cdot\left\{\omega_{i}\right\}_{\text {Base }}\right) . \\
\mathbf{F}_{\mathrm{q}}=\sum_{i=1}^{n_{\mathrm{B}}}\left(\left[\mathbf{J}_{S, i}\right]_{\text {Base }}^{T} \cdot\left\{\mathbf{R}_{i}\right\}_{\text {Base }}+\left[\mathbf{J}_{\omega, i}\right]_{\text {Base }}^{T} \cdot\left\{\mathbf{M}_{G, i}\right\}_{\text {Base }}\right),
\end{gathered}
$$

where the subscript Base refers to the base frame, $\left[\mathbf{J}_{S, i}\right]_{\text {Base }}$ and $\left[\mathbf{J}_{\omega, i}\right]_{\text {Base }}$ are the translational and rotational Jacobian matrices of body $i$ expressed in the base frame.

To model the gearbox flexibility, unactuated degrees-of-freedom are introduced at the articulation, allowing a deflection introducing elastic and damping action/reaction torques on the bodies on either side of the articulation. The torques between body $i$ and $j$ are expressed by

$$
\begin{aligned}
& \left\{\mathrm{M}_{G, i}\right\}_{\text {Base }}=-\left(k_{q_{u, l}} \cdot q_{u, l}+d_{q_{u, l}} \cdot \dot{q}_{u, l}\right) \cdot \mathbf{R}_{\text {Base }, i} \cdot \mathrm{u}_{l} \quad \text { (action), } \\
& \left\{\mathrm{M}_{G, j}\right\}_{\text {Base }}=\left(k_{q_{u, l}} \cdot q_{u, l}+d_{q_{u, l}} \cdot \dot{q}_{u, l}\right) \cdot \mathbf{R}_{\text {Base }, i} \cdot \mathbf{u}_{l} \quad \text { (reaction) }
\end{aligned}
$$

where $k_{q_{u, l}}$ and $d_{q_{u, l}}$ are respectively the torsional stiffness and damping of the articulation along local axis $l$.

The machining forces are computed considering the mechanistic approach [14]. Theses forces are computed as the sum of elementary contributions along the tool axis as follows:

$$
\mathrm{F}_{\alpha}=\sum_{k=1}^{n_{s}} K_{\alpha, c} \cdot h \cdot d z+K_{\alpha, e} \cdot d s
$$

with $h$ the uncut chip thickness, $d z$ the height of the elementary slice, $n_{s}$ the number of tool slices [14], $\alpha=t, r, a$ the tangential, radial and axial directions respectively, $K_{\alpha, c}$ and $K_{\alpha, e}$ the cutting coefficients and $d s$ the local cutter edge length. The forces are then applied on the TCP. Their computation is the result of the coupling of two simulators, on the one hand EasyDyn, an in-house multi-body solver and on the other hand DyStaMill, an in-house solver as well, dedicated to the simulation of machining operations [13]. 
The global block diagram of the correction is shown in Fig. 1. The system is controlled with an inverse dynamic controller [15] in which $\mathbf{M}_{\mathbf{q}}(\mathbf{q}), \mathbf{h}_{\mathbf{q}}(\mathbf{q}, \dot{\mathbf{q}}), \mathbf{F}_{\mathbf{q}}$ are computed with the rigid model of the robot in order to provide the assumption that the robot controller is not aware of the flexibility. The Cartesian trajectory $\left[p_{t} \dot{p}_{t} \ddot{p}_{t}\right]^{T}$ is first transposed in the joint space $\left[q_{t} \dot{q}_{t} \ddot{q}_{t}\right]^{T}$ with second order inverse dynamics, also based on a hypothetical perfectly rigid robot, and tracked with the controller generating the motor torques $\left[u_{1, t} \ldots u_{n_{\text {dof }}, t}\right]^{T}$. The aim of this architecture is to reproduce the conditions where the inner controller of the robot is not accessible and the input is the trajectory sent to the system.

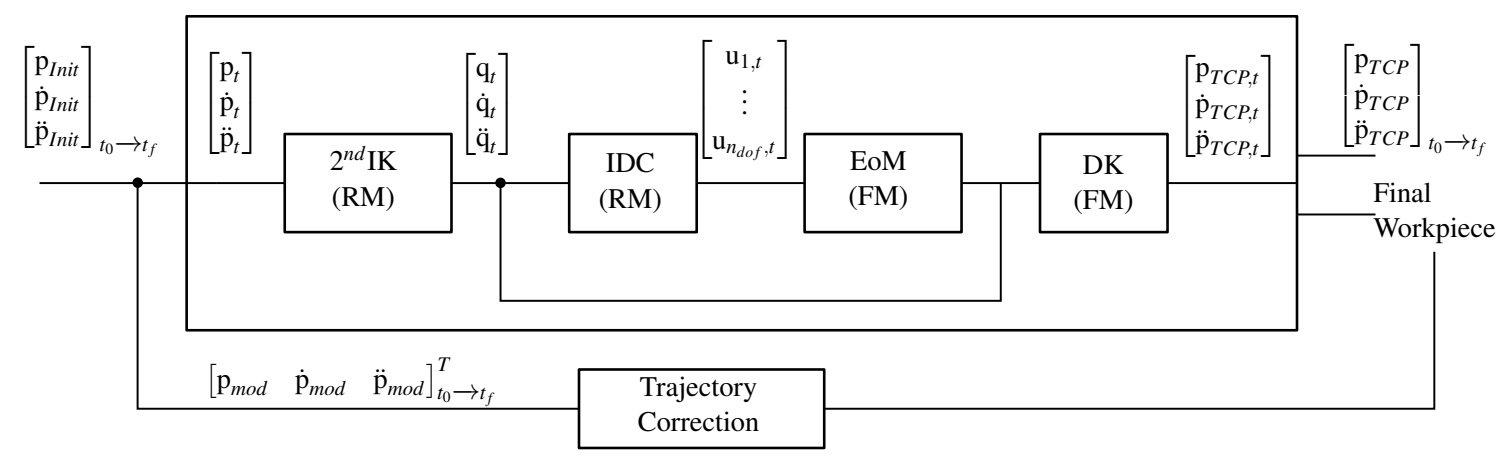

Figure 1: Block diagram of the off-line operational trajectory correction. With IK: Inverse Kinematics, IDC: Inverse Dynamics Controller, EoM: Equations of Motion, DK: Direct Kinematics, RM: Rigid model, FM: Flexible Model. The initial trajectory based on the G-code is $\left[p_{\text {Init }} \dot{p}_{\text {Init }} \ddot{p}_{\text {Init }}\right]_{t_{0}}^{T} \rightarrow t_{f}$. The simulated TCP position is $\left[p_{T C P} \dot{p}_{T C P} \ddot{p}_{T C P}\right]_{t_{0}}^{T} \rightarrow t_{f}$ and the corrected trajectory $\left[p_{\text {mod }} \dot{p}_{\text {mod }} \ddot{p}_{\text {mod }}\right]_{t_{0}}^{T} \rightarrow t_{f}$.

\section{DEVIATIONS COMPENSATION}

The trajectory is discretized by nodes $H_{i}$ between which an Hermite interpolation is carried out. Each node includes a homogeneous transformation matrix and velocity requirements for the TCP.

$$
H_{i}=\left[\begin{array}{cc}
R_{n, i} & p_{n, i} \\
0 & 1
\end{array}\right], \quad \dot{H}_{i}=\left[\begin{array}{ll}
\omega_{n, i} & v_{n, i}
\end{array}\right]
$$

where $R_{n, i}$ the rotation matrix of node $i$ whose $z$ axis correspond to the tool orientation, $p_{n, i}$ the TCP position, $\omega_{n, i}$ and $v_{n, i}$ the rotational and translational velocities respectively.

The aim is to model the trajectory using a minimal number of nodes. The first step of the compensation method is to detect the areas where the deviation is problematic in order to act on the existing nodes position to reduce the machining error and possibly place additional nodes. A first correction is carried out using the mirroring approach for each node, which consists in applying at the trajectory nodes the error between the actual TCP position and the node position. This correction intends to compensate mainly the deviations from static forces, such as the impact of gravity on the structure.

Afterwards, the operation is simulated to determine the deviation at each time step. A correction is then applied on the nodes having an impact on the machining, i.e. the nodes surrounding trajectory sections where the robot is milling. The updated trajectory is the input for the following simulation. This correction is computed as the mean machining error caused for the upcoming path section, meaning that the node $i$ is replaced depending on the machining error accumulated in path section $i \rightarrow i+1$. The deviations of a dynamical nature are thus taken into account since this error is computed with the system response from the integration in time of the equation of motions. The trajectory $\mathbf{H}$ is updated at each iteration $k$ with the modified position of the nodes $\mathbf{H}_{\text {mod. }}$. It allows to anticipate the deviation, in particular for the entry, and accept to deviate from the ideal trajectory while the TCP is outside the matter if it is in the interest of reducing the machining error. 
Once the nodes have been repositioned at best, the global machining error is compared to a threshold and if the gap is still too important, a node is added in the critical position, being the location presenting the highest deviation. Finally, when the error is under the threshold, the trajectory is sent to the optimization layer where, in addition to the position, the velocity at each of the nodes will be considered as a design variable. The node-repositioning algorithm is presented in Fig. 2.

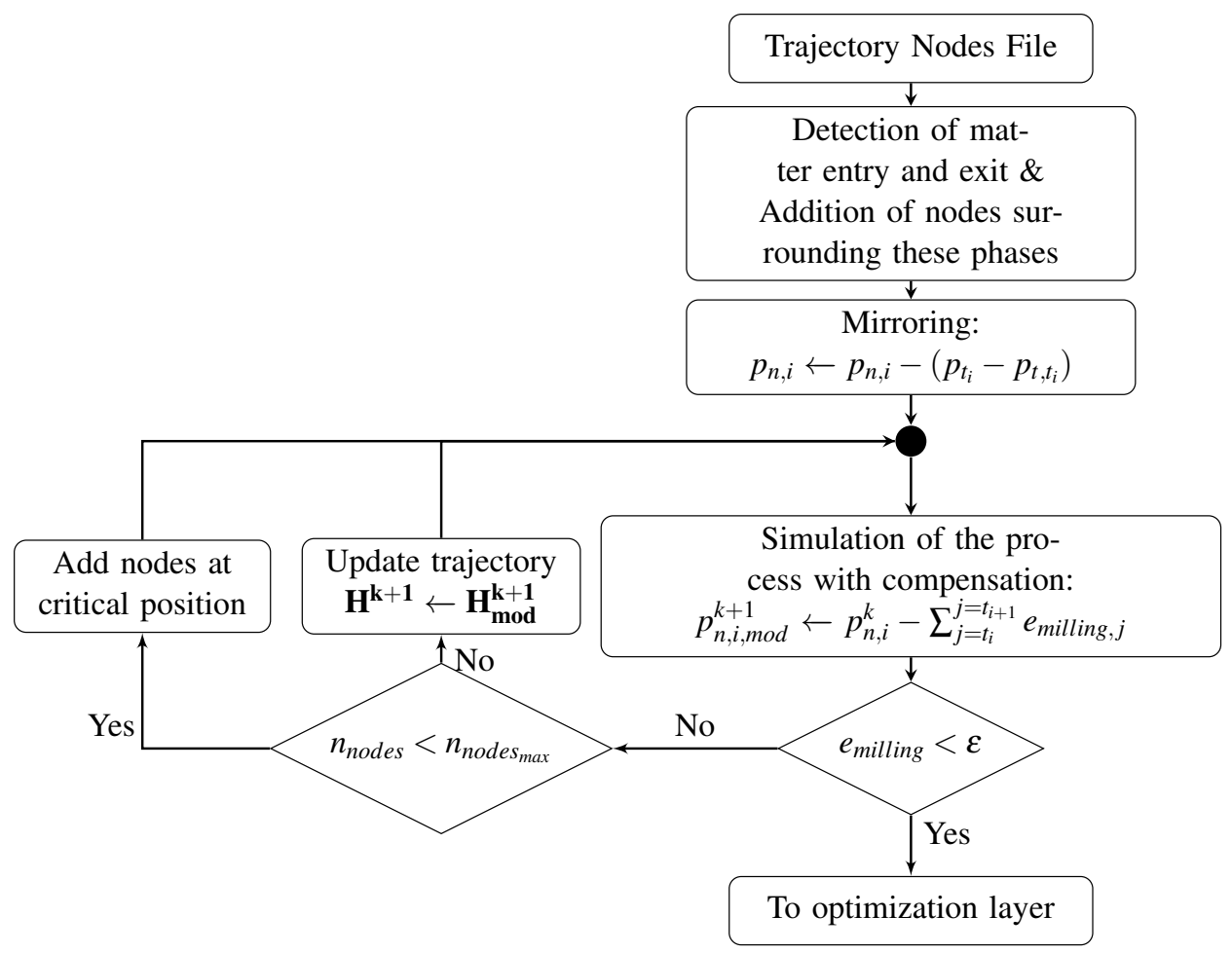

Figure 2: Trajectory nodes repositioning algorithm.

\section{TRAJECTORY NODES OPTIMIZATION}

Once the nodes have been replaced based on successive simulations of the dynamical system, a finer result can be obtained by acting on the velocity $v_{n, i}$ as well as the position $p_{n, i}$ of the nodes linked to the machining. The optimization problem is given in the following equation:

$$
\min _{\mathrm{p}_{n, i}, \mathrm{v}_{n, i}, \mathrm{p}_{n, i+1}, \mathrm{v}_{n, i+1}} \cos t=\sum_{k=0}^{N}\left\|\mathrm{p}_{T C P}-\mathrm{p}_{t}\right\|_{2}^{2} \cdot \gamma_{k}
$$

where the parameters are successive nodes $\left(n_{i}, n_{i+1}\right)$ positions and velocities, $p_{T C P}$ is the tool center point position, $p_{t}$ the ideal path and $\gamma_{k}$ is a weighting factor equal to 1 while machining and 0 else where and $N$ is the number of observation points, i.e. the number of time steps to simulate the process from $t_{0}$ to $t_{f}$. In order to reduce the design variables research horizon and remain in feasible areas, boundaries $\left[x_{l} ; x_{u}\right]$ are imposed by logarithmic barrier transformation $\operatorname{tr}(x)$ as presented in Eq. 9. As stated before, a deterministic method is preferred, hence quasi-Newtonian 1-BFGS is selected.

$$
\begin{array}{r}
\operatorname{tr}(x)=\ln \left(x-x_{l}\right)-\ln \left(x_{u}-x\right) \\
t^{-1}(y)=\frac{e^{y} x_{u}+x_{l}}{1+e^{y}}
\end{array}
$$

For the sake of readability, the legend of the curves used in Figs. 4a,5,7a,8 and 9b is detailed and kept along the paper. The red dashed line with circles (- - - ) represents the trajectory sent to the system and the without symbol one (-- ) gives the system response. The blue dashed line with triangles $(-\cdot-\cdot)$ shows the trajectory after the node repositioning algorithm and the corresponding 
tool motion without symbols (---) .The final compensated trajectory sent to system is presented with the green dashed line with squares (- - ) and the full line (-) represent the final tool motion. Finally, the dotted line (…) line shows the moments the tool enters and leaves the matter.

The machining operation performed by the aforementioned systems is the face milling of a Al6060 block with a flat-end mill of diameter $D_{\text {tool }}=10 \mathrm{~mm}$ with 2 teeth presenting a $30^{\circ}$ helix angle in half-immersion over a cutting dept of $2 \mathrm{~mm}$. The spindle rotation speed is $11250 \mathrm{RPM}$ for a feed per tooth of $0.13 \mathrm{~mm} /$ tooth. The trajectory is limited to a single pass along the $y$ axis. The nodes added are then the ones surrounding the entry and exit and one at the middle of the part (since the section with the highest error is between entry and exit).

\subsection{Tool mass system}

In the first instance, the node replacement with optimization layer has been carried out for the simplified tool-mass model represented in Fig. 3. This system is composed of a mass connected to a support with a pair of spring/damper along the $x$ and $y$ directions. The motion of the support is imposed by the trajectory. The inertia parameters as well as stiffness and damping values were chosen such as the tool-mass exhibits a rather similar deflection behavior than the TX200 for this kind of machining operations which results in $M=200 \mathrm{~kg}, k=100 \mathrm{kN} / \mathrm{m}$ and $c=4 \mathrm{kN} . \mathrm{s} / \mathrm{m}$.

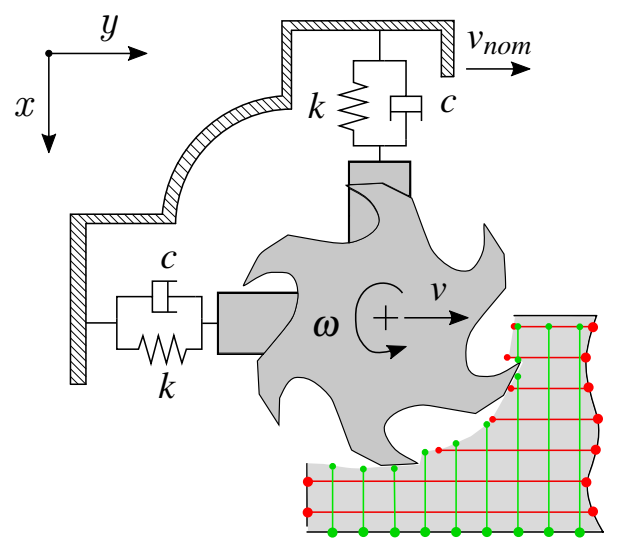

Figure 3: Simplified system, consisting of a tool attached, through spring-damper pairs, to a support moving along $y$ direction and machining a workpiece in half-immersion.

The result of the repositioning algorithm and the optimization of the nodes positions and velocities is presented in Fig. 4a, where the amplitude of the error is already strongly reduced in the milling area with the first compensation stage (- - ). The optimization layer managed to reduce the amplitude of the gap at the entry and exit as shown in Fig. 5. The evolution of the cost function along the corrections is displayed in Fig. 4b. The optimization layer does not bring strong improvement since its contribution is mainly located around the entry and exit.

\subsection{Two degree of freedom flexible system}

The second system implemented is a two-degree-of-freedom system with additional flexibility at the articulations presented in Fig. 6. This model introduces coupling between $q_{1}$ and $q_{2}$ and nonlinearity between the task space and the operational space since the actuated degrees of freedom are the joints angular positions. The first body $\left(s_{1}\right)$ is linked to the ground through the motor where the gearbox flexibility is modeled by a torsional spring-damper pair (Eq. 5). The motor actuating the second link $s_{2}$ is located at the tip of $s_{1}$, with the gearbox flexibility modeled in the same way. The unactuated degrees-of-freedom representing the articular deflection are gathered in $\mathrm{q}_{u}=\left[\begin{array}{ll}q_{3} & q_{4}\end{array}\right]^{T}$. An additional mass $s_{3}$ is placed at the tip of the body $s_{2}$ in order to represent the equivalent charge of the spindle. Just as the tool-mass model, the machining operation is the 


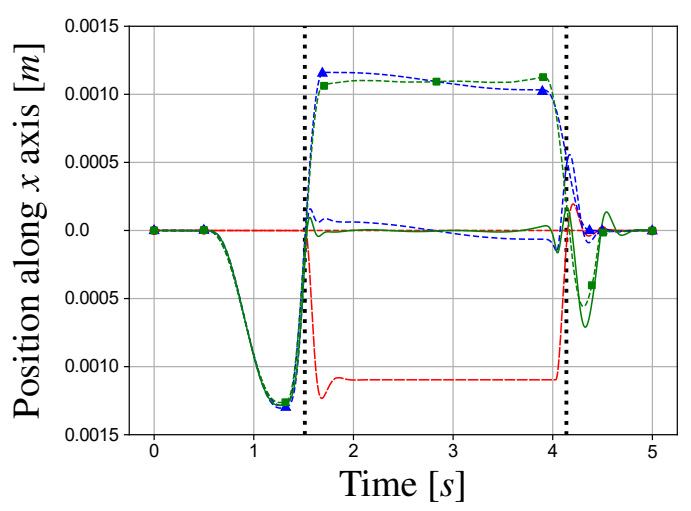

(a)

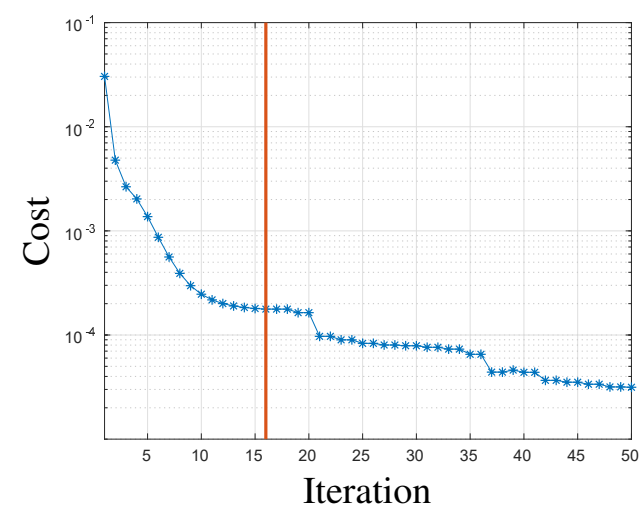

(b)

Figure 4: (a) Evolution of the tool trajectories along $x$ direction throughout the correction procedure for the tool-mass system. Legend is given in Sec. 4. (b) Evolution of the cost function. The line (-) symbolize the demarcation between the node repositioning and the optimization layer.

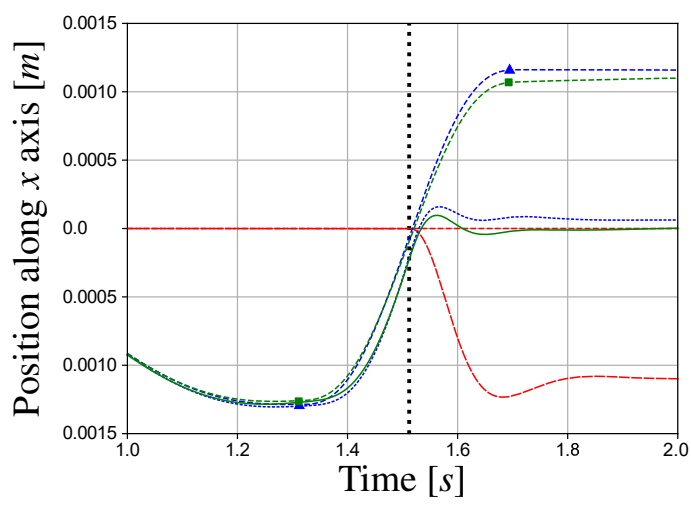

(a)

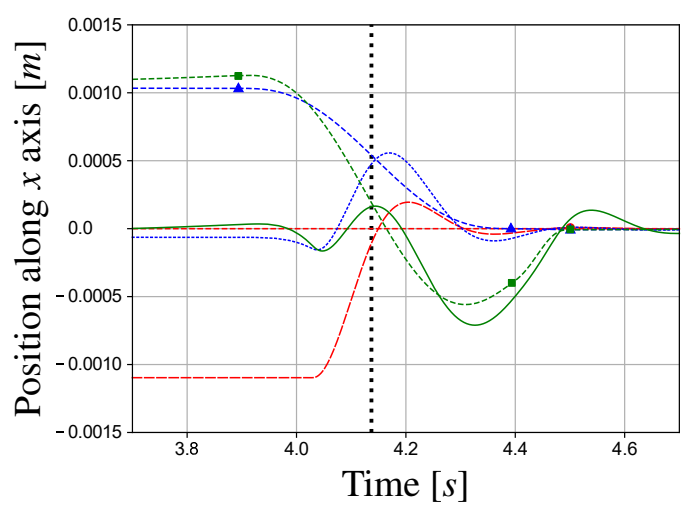

(b)

Figure 5: Close-up views of the trajectories for the tool-mass system presented in Fig. 4a for entry (a) and exit (b) of matter.

surface milling in half-immersion of the tool along the $y$ axis. The inertia properties are chosen to behave the same way as the machining robot does.

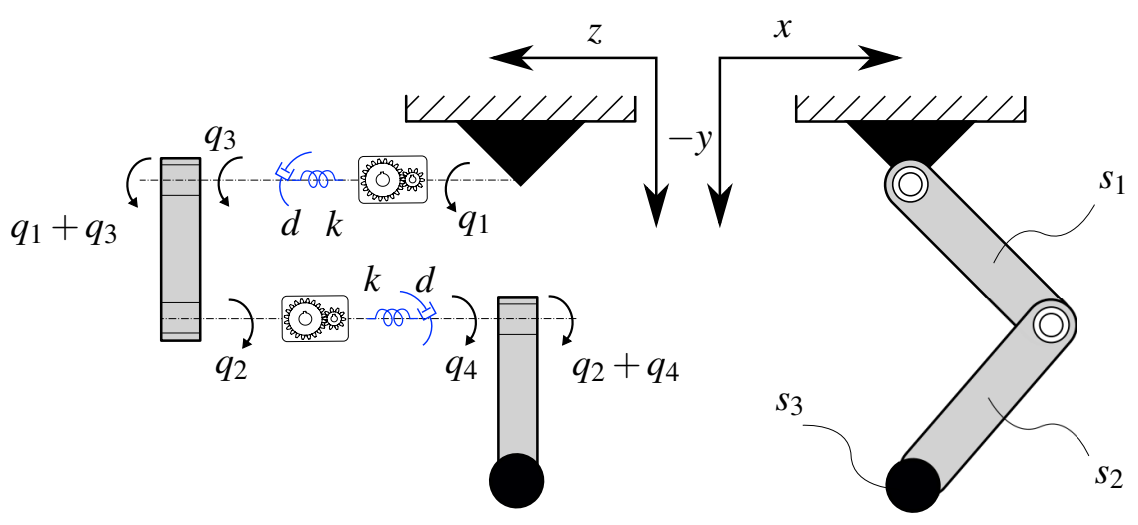

Figure 6: Two actuated degrees-of-freedom system with articular flexibility. Solids $s_{1}$ and $s_{2}$ are the robot links and $s_{3}$ is a mass supporting the tool. The articular flexibility is represented by the deflections $q_{3}$ and $q_{4}$ with the torsional springs and dampers $k$ and $d$. 
Similarly to the tool-mass model, the tracking error is reduced within the machining part of the trajectory. The compensated and final trajectories are shown in Fig. 7a and the evolution of the cost value in Fig. 7b. The anticipation of the deviation at the entry and exit resulting from the optimization layer is emphasized in Fig. 8.

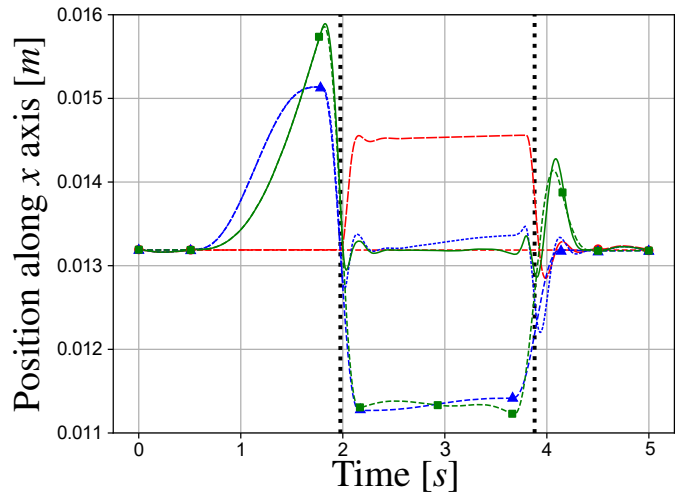

(a)

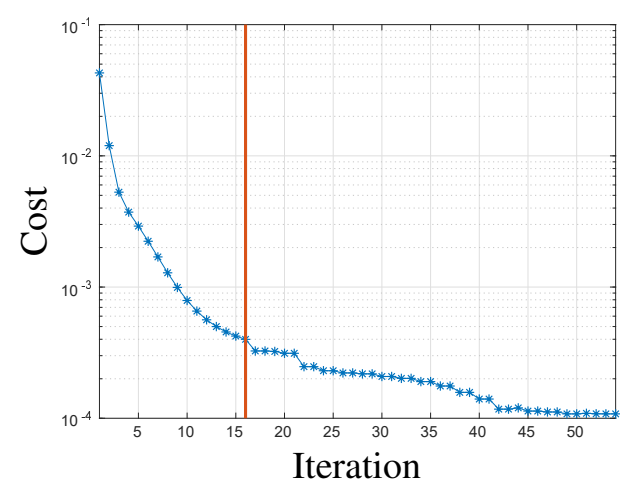

(b)

Figure 7: (a) Evolution of the tool trajectories along $x$ direction throughout the correction procedure for the two-degree-of-freedom system. Legend is given in Sec. 4. (b) Evolution of the cost function for the trajectory correction of the two-degree-of-freedom system. The line (-) symbolize the demarcation between the node repositioning and the optimization layer.

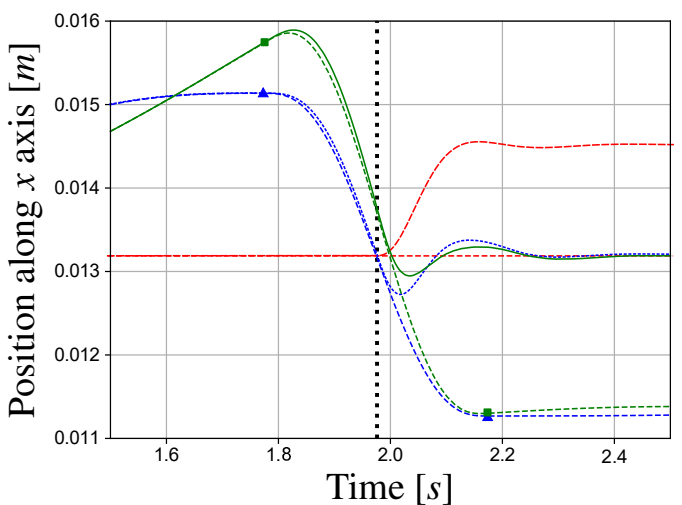

(a)

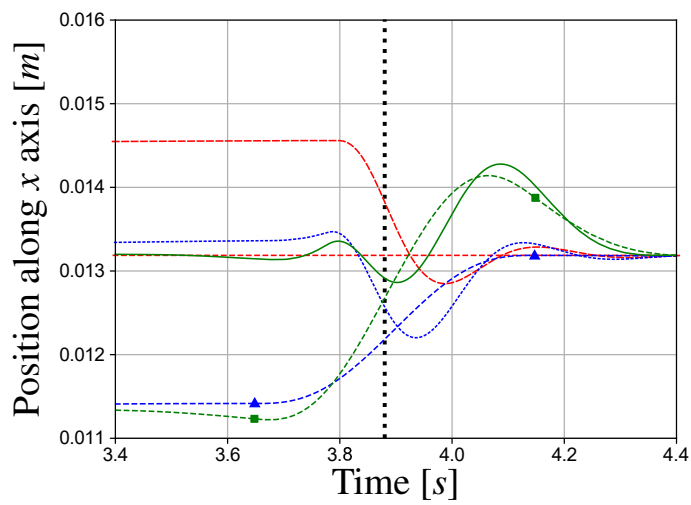

(b)

Figure 8: Close-up views of the trajectories presented in Fig. 4a for entry and exit of matter.

\subsection{Anthropomorphic Robot}

The anthropomorphic robot studied in this work is the TX200 from Stäubli illustrated in Fig.9a. The multibody system includes is the result of the complete identification of the flexibility, with the tri-axial torsional flexibility model [3]. Additional degrees of freedom are then induced at the articulations and links, leading to a multiple-degrees-of-freedom system composed of six actuated degrees-of-freedom $\mathrm{q}_{a}=\left[q_{a, 1} \ldots q_{a, 6}\right]$ and 34 others $q_{u}$, modeling the deflections [16]. The triaxial method consists in three torsional spring/damper pairs consecutively connected after the gearbox and oriented along each of the three local frame directions. The unactuated degrees-offreedom at the second joint $q_{u, 2}\left(q_{x, 2}, q_{y, 2}, q_{z, 2}\right)$ thus represent the joint deflections and lead to the following formulation of the Body 2 center of mass $\left(G_{\text {Body }} 2\right)$ location with respect to the base 
$\left(O_{\text {Base }}\right)$ on which the robot is mounted

$$
\begin{aligned}
& \mathbf{H}_{O_{\text {Base }}, G_{\text {Body } 2}}=\mathbf{H}_{O_{\text {Base }}, O_{\text {Body } 2}} \cdot \mathbf{H}_{\text {rotz }}\left(q_{a, 2}\right) \cdot \mathbf{H}_{\text {rotz }}\left(q_{z, 2}\right) \cdot
\end{aligned}
$$

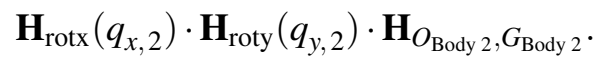

Similarly to the two-degree-of-freedom planar robot detailed in Section 4.2, the rigid model of the robot is used for the inverse dynamics controller as well as the second order inverse kinematics (Fig. 1). As well as the previous systems, the robot is machining along the $y$ direction, maintaining the $x$ position constant. The evolution of trajectories along the $x$ direction is presented in Fig. 9b. At the exit, the dynamic deflection is well compensated however the contribution of the optimization for the entry is rather small. It can be improved by changing the weighting of the entry section in the definition of the cost function.

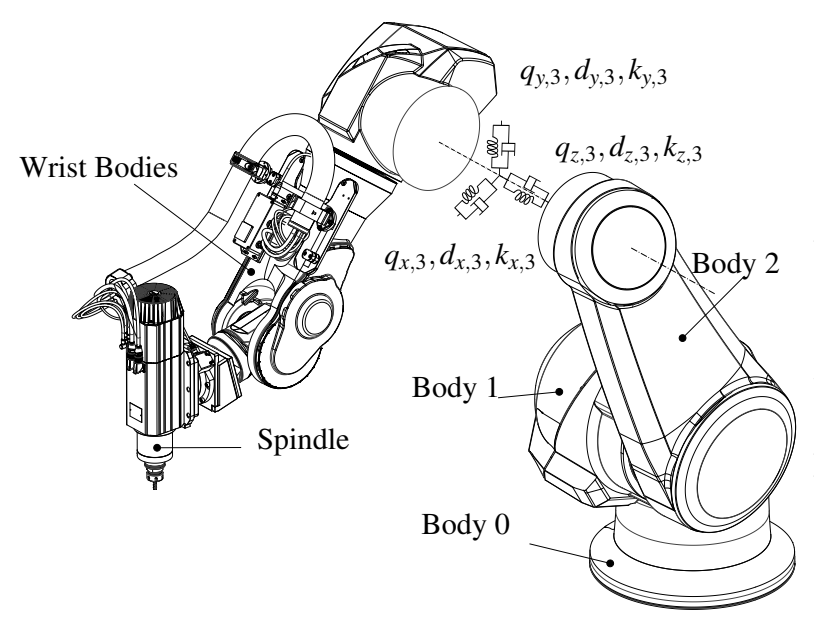

(a)

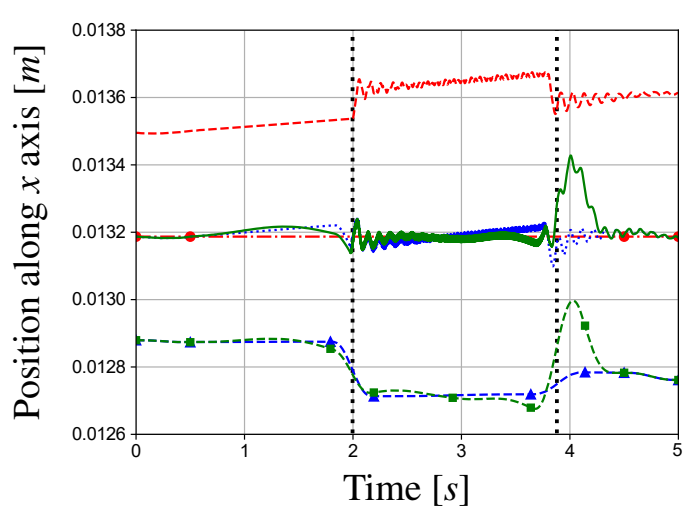

(b)

Figure 9: (a) Representation of the multibody modeling of a Stäubli TX200 robot dedicated to machining. For readability reasons, the articular flexibility is only explicitly shown for the third joint. (b) Evolution of the tool trajectories along $x$ direction throughout the correction procedure for the TX200 robot model. Legend is given in Sec. 4.

\section{PERSPECTIVES}

As a perspective of development, a stronger contribution can be given to the optimization part. A refinement of the cost function is to be considered, where, instead of using the accumulated positioning error obtained by the comparison of trajectories, the difference between the ideal workpiece (in a dexel form) and the workpiece resulting from the simulation is proposed. The importance is centered on the realization of the expected workpiece and no longer on the accomplishment of a trajectory. Since the machining is preferred at constant velocity for stability and surface quality reasons, a penalty in the norm of the TCP velocity may be added. The improved cost function is presented as follows :

$$
J=\sum_{i=0}^{N_{x}} d_{\Delta, x, i}+\sum_{j=0}^{N_{y}} d_{\Delta, y, j}+\sum_{k=0}^{N_{z}} d_{\Delta, z, k}+\sum_{l=0}^{N_{t}} \gamma_{l} \cdot\left\|\mathrm{v}_{T C P, s_{l}}-\mathrm{v}_{T C P, t_{l}}\right\| \cdot d t
$$

where $d_{\Delta, x, i}$ represents the difference between the dexel $i$ along the $x$ axis obtained from the simulation with the corresponding one from the ideal workpiece. The illustration of the difference between dexels workpiece in given in Figure 10. The time code of the trajectory nodes can be included in the design variables set in order to enable the nodes to be more moving.

Furthermore, a balance between the two correction layers (node-repositioning/introduction and optimization) to define an optimal amount of nodes to be added for a sufficient pre-compensation. 


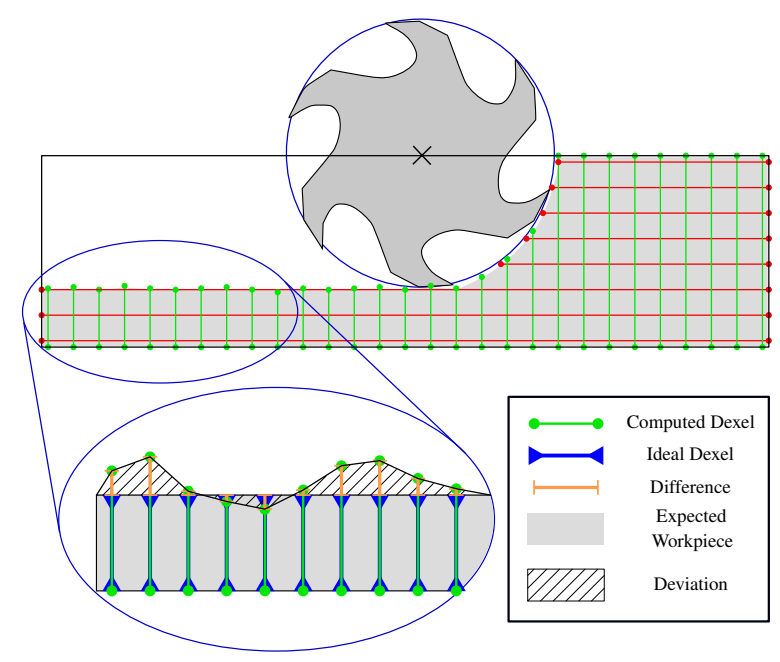

Figure 10: Determination of difference between the ideal workpiece and the resulting workpiece from the dynamic simulation.

Finally, further investigation and tests are being considered for complex multibody systems, such as the TX200 robot model, to challenge the proposed method. For such applied case, experimental validation is planned. The dexel approach opens the perspective of more complex trajectories to be simulated [17], the deployment of the method is intended for 5-axis operations.

\section{CONCLUSIONS}

Robotic machining is a growing technology but suffers from the lack of accuracy for hard-material machining, mainly caused by the overall structure flexibility. An in-dept studies of the phenomena allowed to model the flexibilities. Using these models, it is then possible to estimate, up to a certain accuracy, the deflections at the tool-tip caused by the machining forces. Within the frame of Industry 4.0, these models are virtual twins of the operation, and can be used to predict the behavior of the robot as well as the result. The machine models can be expressed as under-actuated multibody systems, where some degrees-of-freedom are the articulations and others represents deflections.

This article presents a method taking advantage of such models to build a tool trajectory, expressed in the operational space, anticipating the deviations from static and dynamic nature while subjected to machining forces. The method is employed on under-actuated multibody systems of increasing complexity with flexibility. The reduction of the deviation can be improved by varying the cost function as well as the amount of added nodes. For more complex systems as the one presented, the simulation time becomes a constraint since the optimization layer requires several iterates to propose improvements.

\section{ACKNOWLEDGMENTS}

The authors would like to acknowledge the Belgian National Fund for Scientific Research (FNRSFRS) for the grant allotted to V. Dambly.

\section{REFERENCES}

[1] Kim, S.H., Nam, E., Ha, T., Hwang, S.H., Lee, J., Park, S.h., Min, B.K.: Robotic machining: A review of recent progress. International Journal of Precision Engineering and Manufacturing 20 (08 2019)

[2] Verl, A., Valente, A., Melkote, S., Brecher, C., Ozturk, E., Tunc, T.: Robots in machining. CIRP Annals (06 2019) 
[3] Huynh, H.N., Assadi, H., Dambly, V., Rivière-Lorphèvre, E., Verlinden, O.: Direct method for updating flexible multibody systems applied to a milling robot. Robotics and ComputerIntegrated Manufacturing 68 (04 2021)

[4] Reinl, C., Friedmann, M., Bauer, J., Pischan, M., Abele, E., Von Stryk, O.: Model-based offline compensation of path deviation for industrial robots in milling applications. IEEE/ASME International Conference on Advanced Intelligent Mechatronics, AIM (07 2011) 367-372

[5] Klimchik, A., Bondarenko, D., Pashkevich, A., Briot, S., Furet, B.: Compliance error compensation in robotic-based milling. Informatics in Control, Automation and Robotics $\mathbf{2 8 3}$ (09 2014)

[6] Zaeh, M., Schnoes, F., Obst, B., Hartmann, D.: Combined offline simulation and online adaptation approach for the accuracy improvement of milling robots. CIRP Annals 69 (05 2020)

[7] Mamedov, S., Popov, D., Mikhel, S., Klimchik, A.: Compliance error compensation based on reduced model for industrial robots. 15th International Conference on Informatics in Control, Automation and Robotics (01 2018) 180-191

[8] Kaldestad, K., Hovland, G.: Off-line path correction of robotic face milling using static tool force and robot stiffness. (09 2015) 5506-5511

[9] Schnoes, F., Zaeh, M.: Model-based planning of machining operations for industrial robots. Procedia CIRP 82 (01 2019) 497-502

[10] Cordes, M., Hintze, W.: Offline simulation of path deviation due to joint compliance and hysteresis for robot machining. The International Journal of Advanced Manufacturing Technology 90 (04 2017)

[11] Brüls, O., Bastos, G.J., Seifried, R.: A Stable Inversion Method for Feedforward Control of Constrained Flexible Multibody Systems. Journal of Computational and Nonlinear Dynamics 9(1) (10 2013) 011014.

[12] Altintas, Y., Kersting, P., Biermann, D., Budak, E., Denkena, B., Lazoglu, I.: Virtual process systems for part machining operations. CIRP Annals - Manufacturing Technology 63 (12 2014)

[13] Huynh, H.N., Rivière-Lorphèvre, E., Ducobu, F., Ozcan, A., Verlinden, O.: Dystamill: a framework dedicated to the dynamic simulation of milling operations for stability assessment. The International Journal of Advanced Manufacturing Technology 98(5) (2018) 21092126

[14] Altintas, Y., Engin, S.: Generalized modeling of mechanics and dynamics of milling cutters. Cirp Annals-manufacturing Technology - CIRP ANN-MANUF TECHNOL 50 (12 2001) 25-30

[15] Siciliano, B., Sciavicco, L., Luigi, V., Oriolo, G.: Robotics: Modelling, Planning and Control. (01 2009)

[16] Hoai Nam, H.: Robotic machining: Development and validation of a numerical model of robotic milling to optimise the cutting parameters. PhD thesis, University of Mons (09 2019)

[17] Dambly, V., Huynh, H.N., Verlinden, O., Rivière-Lorphèvre, E.: Development of tri-dexel based cutting simulator for cutter-workpiece engagement and cutting forces determination. (06 2021) 399-402 